Military Technical College Kobry El-Kobbah, Cairo, Egypt

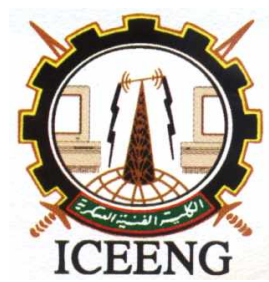

\author{
$6^{\text {th }}$ International Conference \\ on Electrical Engineering \\ ICEENG 2008
}

\title{
MRC Revisited: A reduced complexity Antenna diversity scheme
}

$$
\text { By }
$$

\author{
Yahya Z. Mohasseb*
}

\section{Abstract:}

This paper presents a modification of the Maximum Ratio Combining (MRC) technique used in antenna diversity systems. The modified scheme requires only one RF down conversion chain and one matched filter for the whole system and therefore provides significant complexity reduction. The bandpass equivalent of the conventional baseband MRC scheme is derived first, and a modification is proposed to attain a practical implementation. Simulation results for different modulation schemes verify the validity of the proposed model and show no loss of performance with respect to the derived bandpass scheme.

\section{Keywords:}

Diversity reception, Wireless Channels, Maximum Ratio Combining, MRC, Raleigh Fading. 


\section{Introduction:}

Several forms of diversity are often used in wireless networks to combat the effects of fading. The basic concept is to transmit the signal over several dimensions; time, space, frequency or any combination of them, to increase the reliability of the received signal. In space, diversity can be achieved on the receive side or, on the transmit side e.g. [1-2]. Receive antenna diversity makes use of a number of well separated receive antennas. The independent receptions from each one are combined either by selecting the strongest signal; i.e. selection diversity [3], by choosing an alternate antenna if the signal falls below a threshold; i.e. switched diversity [15], or by linear combination of the received signals. The latter is called maximum ratio combining (MRC). Of late, a new form of diversity, called cooperative diversity has also been proposed [4-6]; where multiple single antenna nodes are used as a virtual antenna array to relay the signal for one active source/destination pair.

Receive antenna diversity, with Maximum Ratio Combining (MRC), is perhaps the oldest form of diversity found in literature [7]. However, due to its wide spread use, it continues to receive ample attention. In particular, there is continuing interest in quantifying its performance in a variety of operating conditions. For example, in [8], a theoretical method was developed to analyze MRC receivers using BPSK and M-PSK modulation in spatially correlated Rayleigh fading channels, with perfect and imperfect channel knowledge; whereas in [9-10] performance of a binary modulated signal was studied for an MRC receiver with correlated diversity branches based on imperfect channel state information both in white and colored noise respectively.

This continuing interest in MRC is driven by the fact that it can be implemented in preexisting systems without the need to modify the transmission scenario. It is also suitable for uplinks in base stations, where it is usually impractical for the mobile unit to have multiple transmit antennas to afford MIMO-Space Time Coding Schemes. However, the main disadvantage of MRC is the fact that it requires complete RF chains. Attempts to solve this problem in a hybrid transmit antenna selection/maximum-ratio combining scheme was made in [11] where a reduced subset of the transmitting antennas are selected and the number of required RF chains at the receiver is reduced accordingly. In this paper, a method to reduce the number of RF down converters needed in a receive diversity system is derived. It is shown that Maximum Ratio Combining (MRC) can be implemented on the bandpass RF signal and prior to the matched filter. This results in a significant hardware reduction at the receiver due to the use of a single RF down converter and a single matched filter. The exact banpass equivalent of the MRC scheme is first derived. It is shown that such a scheme requires a $90^{\circ}$ phase shift of the incoming RF signal in each diversity branch. In order to avoid the carrier phase estimation for 
each branch, an approximate equation for the modified MRC scheme is derived; which requires only the use of a delay line. The approximations used to arrive at the modified MRC scheme are well justified analytically and are also validated by simulations for different types of modulations and different diversity orders. The results show no loss of performance compared to the conventional MRC scheme which requires $L$ RF down converters and $L$ matched filters.

Section 2 presents the system model and quickly reviews the conventional MRC scheme. In doing so, emphasis is made on expressing all signals in their respective bandpass forms. In Section 3, this bandpass representation is used to derive the equivalent MRC scheme prior to RF down conversion and matched filtering. In Section 4 , the derived equivalent scheme is simplified to ensure a feasible implementation. These simplifications result in an approximate MRC scheme. Section 5 validates the approximations made and quantifies the proposed scheme. Finally, Section 6 presents the conclusions.

\section{$\underline{\text { 2.The system Model }}$}

In this section conventional maximum ratio combining (MRC) is briefly reviewed. The goal of this brief review is two fold; first to allow comparison with the proposed scheme by providing the real bandpass representation of the MRC scheme which is traditionally represented in complex baseband instead. Second, to motivate the proposed RF MRC scheme by showing that a simple exchange of matched filtering and frequency down conversion is not sufficient.

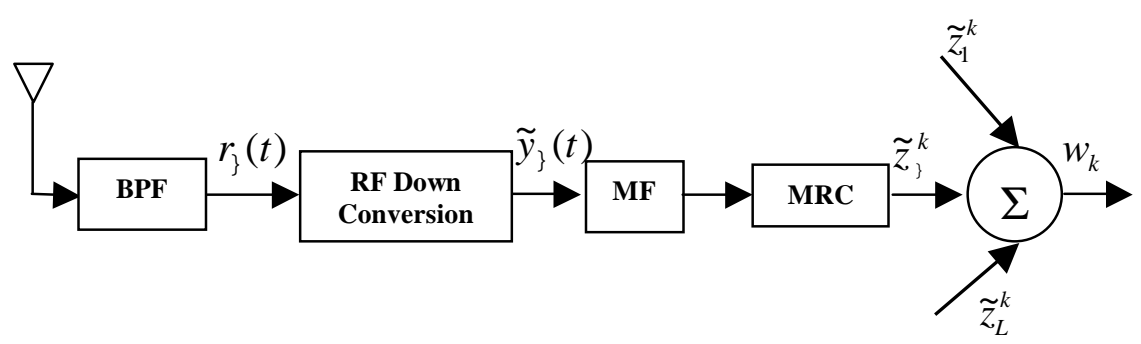

Figure (1): Block Diagram of the conventional MRC scheme.

Consider a system with a single transmit antenna and $L$ receive antennas. The complex baseband transmitted signal $g(t)$ can be expressed as :

$g(t)=\sum_{k=0}^{\infty} d_{k} p\left(t-k T_{s}\right)$ 


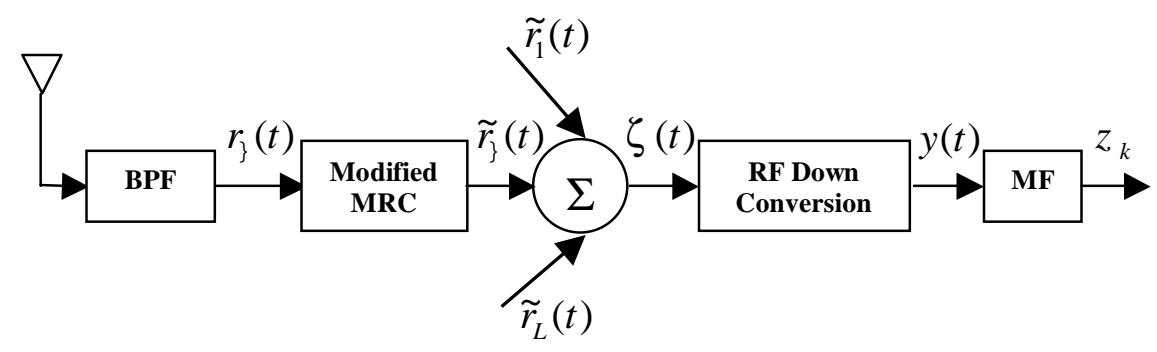

Figure (2): Block Diagram of the proposed RFMRC scheme.

Where $d_{k}$ is the $k^{\text {th }}$ data symbol, $p(t)$ is the pulse shaping function, e.g. the Square Root Raised cosine pulse shape [12]. Following frequency up-conversion, the real valued bandpass signal transmitted through the antenna can be expressed as:

$$
s(t)=\mathfrak{R}\left\{g(t) e^{j w_{c} t}\right\}=\Re\left\{\sum_{k=0}^{\infty} d_{k} p\left(t-k T_{s}\right) e^{j w_{c} t}\right\}
$$

Where $\Re\{\cdot\}$ denoted the real part and $w_{c}$ is the carrier frequency. Thus, the received signal at the input of antenna I becomes:

$r_{1}(t)=h_{1} s(t)+n_{1}(t)=\Re\left\{\sum_{k=0}^{\infty} d_{k} h_{1} p\left(t-k T_{s}\right) e^{j w_{c} t}\right\}+n_{1}(t)$

where $n_{1}(t)$ is a bandpass RF noise process representing the filtered Additive White Gaussian Noise (AWGN) at the output of the noise limiting bandpass filter. Assuming the width of the spectral density of the bandpass filter is much smaller than the carrier frequency, $n_{1}(t)$ can be represented [12 eq. (4.1-37)] as $n_{1}(t)=\mathfrak{R}\left\{v_{1}(t) e^{j w_{c} t}\right\}$ where $v_{1}(t)$ is the equivalent complex baseband noise process; with zero mean and Power Spectral Density (PSD) equal to $N_{o}$ Therefore, we may rewrite $r_{1}(t)$ in the form:

$r_{1}(t)=h_{1} s(t)+n_{1}(t)=\Re\left\{\left(\sum_{k=0}^{\infty} d_{k} h_{1} p\left(t-k T_{s}\right)+v_{1}(t)\right) e^{j w_{c} t}\right\}$

Conventionally, Maximum Ratio Combining (MRC) is performed after RF down conversion and matched filtering as shown in Figure 1. This necessitates the use of $L$ RF down conversion schemes and $L$ matched filters. The sufficient statistic used to detect the data symbol $d_{k}$ is the $w_{k}$ given by: 


$$
\begin{aligned}
w_{k}= & \sum_{1=1}^{L} z \%_{0}=\sum_{1=1}^{L} h_{1}^{*} \int_{(k-1) T_{s}}^{k T_{s}} y_{1}(\lambda) p\left(T_{s}-t+\lambda\right) d \lambda \\
& =\sum_{1=1}^{L} h_{1}^{*} \int_{(k-1) T_{s}}^{k T_{s}}\left(\sum_{k^{\prime}=0}^{\infty} d_{k^{\prime}} h_{1} p\left(\lambda-k^{\prime} T_{s}\right)+\nu_{1}(\lambda)\right) p\left(T_{s}-t+\lambda\right) d \lambda
\end{aligned}
$$

Assuming $p(t)$ satisfies Nyquist first criterion, and using simple algebra we get:

$w_{k}=\sum_{1=1}^{L}\left|h_{1}\right|^{2} d_{k}+\int_{(k-1) T_{s}}^{k T_{s}} h_{1}^{*} v_{1}(\lambda) p\left(T_{s}-t+\lambda\right) d \lambda=\sum_{1=1}^{L}\left(\left|h_{1}\right|^{2} d_{k}+h_{1}^{*} v_{1}^{k}\right)$

Where $v_{1}^{k}$ is the discrete noise process resulting from sampling the output of the matched filter at each symbol period; which is assumed to be a zero mean uncorrelated Gaussian random variable with $\sigma_{v}^{2}=N_{o} / 2$. Equation (6) represents the conventional MRC receiver. As discussed earlier it uses $L$ RF chains to down convert each diversity branch first.

\section{Deriving the RF equivalent of Conventional MRC}

In the following paragraphs, it is shown that a simple exchange of the RF down conversion block and the matched filter is not sufficient to detect the modulated symbol and that a modification of the MRC scheme is needed. The receiver structure will be considered the same as in Figure 2. However, it is assumed that the MRC is achieved by multiplying the RF signal by the complex conjugate of the channel coefficient. That is, the same MRC block of Figure 1 is used. Our attempt here is to find the RF equivalent of multiplying by the complex conjugate of the channel coefficient in the baseband. In this case the received signal after MRC becomes:

$\hat{r}_{1}(t)=\Re\left\{h_{1}^{*}\left(\sum_{k=0}^{\infty} d_{k} h_{1} p\left(t-k T_{s}\right)+v_{1}(t)\right) e^{j w_{c} t}\right\}$

The notation $\hat{r}_{1}(t)$ is used instead of $r /(t)$ to distinguish this exact equivalent representation from the approximated proposed scheme in the next section.

Since $h_{1}^{*}$ is a complex quantity, Equation (7) can be written as:

$$
\begin{aligned}
\hat{r}_{1}(t)= & \Re\left\{h_{1}^{*}\right\} \mathfrak{R}\left\{\left(\sum_{k=0}^{\infty} d_{k} h_{1} p\left(t-k T_{s}\right)+v_{1}(t)\right) e^{j w_{c} t}\right\} \\
& -\mathfrak{I}\left\{h_{1}^{*}\right\} \mathfrak{I}\left\{\left(\sum_{k=0}^{\infty} d_{k} h_{1} p\left(t-k T_{s}\right)+v_{1}(t)\right) e^{j w_{c} t}\right\}
\end{aligned}
$$

Both the real and imaginary parts of $h_{1}^{*}$ can be obtained via channel estimation. However, the real quantity in the left term is the received real bandpass RF signal $r_{1}(t)$. 
Noting that $\mathfrak{I}\{X\}=\mathfrak{R}\{-j X\}$, the imaginary part may obtained by a $90^{\circ}$ phase shift of $r_{1}(t)$. Thus the RF equivalent of the conventional MRC block is in the form:

$\hat{r}_{1}(t)=\mathfrak{R}\left\{h_{1}^{*}\right\} r_{1}(t)-\mathfrak{I}\left\{h_{1}^{*}\right\} r_{1}(t) e^{\frac{-j \pi}{2}}$

The block diagram representing this equivalent MRC block is depicted in Figure 3-a.

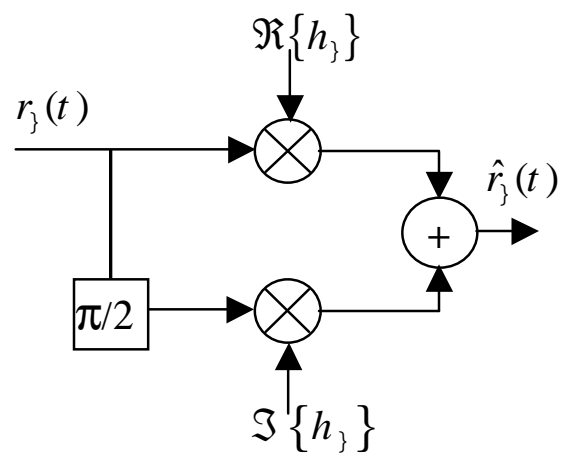

(a) Exact equivalent derived in (9)

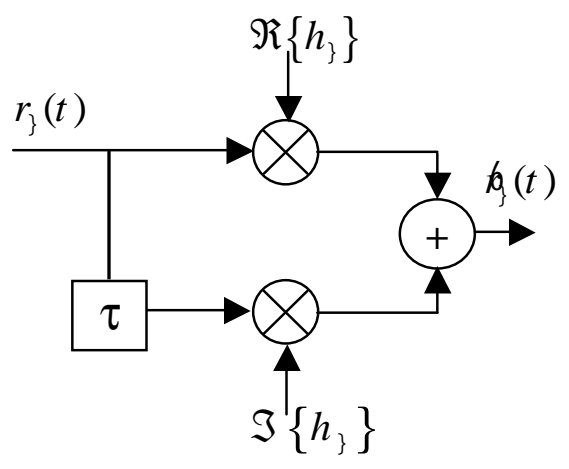

(b) The proposed scheme derived in (10)

Figure 3 Performing MRC on the RF bandpass signal.

\section{The modified MRC scheme}

Although equation (9) derives the exact equivalent of performing MRC on the matched filter output in baseband, it is not practical to assume that the receiver is able to perform a $90^{\circ}$ phase shift over the entire frequency band for an incoming RF signal whose phase is yet to be determined. Indeed, such a phase shift can only be made if a coherent replica of the carrier is made available in each of the $L$ diversity branches. In such case there is no justification to perform the MRC on the bandpass signal since there will be no saving in the RF chains needed.

In order to obtain the $90^{\circ}$ phase shift of the incoming signal without having to employ a coherent carrier, $r_{1}(t)$ is delayed by $\tau$ using a delay line. Referring to Figure 2 and Figure 3-b, the output of the modified MRC block becomes:

$r_{p} /(t)=\Re\left\{h_{1}^{*}\right\} r_{1}(t)-\mathfrak{I}\left\{h_{1}^{*}\right\} r_{1}(t-\tau)$

Where the value of $\tau$ must be chosen such that $\tau=1 / 4 f_{c}$, which results in $w_{c} \tau=2 \pi f_{c} / 4 f_{c}=\pi / 2$. After the summation we have: 


$$
\zeta(t)=\sum_{1=1}^{L}\left(\begin{array}{l}
\Re\left\{h_{1}^{*}\right\} \Re\left\{\left(\sum_{k=0}^{\infty} d_{k} h_{1} p\left(t-k T_{s}\right)+v_{1}(t)\right) e^{j w_{c} t}\right\} \\
-\mathfrak{I}\left\{h_{1}^{*}\right\} \Re\left\{\left(\sum_{k=0}^{\infty} d_{k} h_{1} p\left(t-\tau-k T_{s}\right)+v_{1}(t-\tau)\right) e^{j w_{c} t} e^{-j w_{c} \tau}\right\}
\end{array}\right)
$$

Due to the linearity of the RF down conversion process the signal $y(t)$ becomes:

$$
y(t)=\sum_{i=1}^{L}\left(\begin{array}{c}
\Re\left\{h_{1}^{*}\right\}\left(\sum_{k=0}^{\infty} d_{k} h_{1} p\left(t-k T_{s}\right)+v_{1}(t)\right) \\
\quad+j \mathfrak{I}\left\{h_{1}^{*}\right\}\left(\sum_{k=0}^{\infty} d_{k} h_{1} p\left(t-\tau-k T_{s}\right)+v_{1}(t-\tau)\right)
\end{array}\right)
$$

Following the same procedure as in Equation (5) to obtain the matched filter output at the sampling instant for the $k^{\text {th }}$ symbol we get:

$$
z_{k}=\sum_{1=1}^{L}\left(\begin{array}{c}
h_{1} \Re\left\{h_{1}^{*}\right\} d_{k}+\Re\left\{h_{1}^{*}\right\} v_{1}^{k}+j h_{1} \mathfrak{I}\left\{h_{1}^{*}\right\} d_{k} \rho_{p p}(\tau) \\
+j \mathfrak{I}\left\{h_{1}^{*}\right\}\left(\sum_{i \neq k} h_{1} \rho_{p p}\left(\tau+(k-i) T_{s}\right)+v_{।}^{k, \tau}\right)
\end{array}\right)
$$

Where $\rho_{p p}(\cdot)$ is the autocorrelation function of $p(t)$; which satisfies Nyquist first criterion (e.g. a raised cosine pulse shape). Since $\tau=1 / 4 f_{c} \quad T_{s}$ the following approximations are valid:

$$
\rho_{p p}(\tau) \approx 1 \text { and } \rho_{p p}\left(\tau \pm n T_{s}\right) \approx 0
$$

Hence Equation (13) reduces to:

$$
\begin{aligned}
z_{k} & \approx \sum_{1=1}^{L}\left(h_{1}\left(\mathfrak{R}\left\{h_{1}^{*}\right\}+j \mathfrak{I}\left\{h_{1}^{*}\right\}\right) d_{k}+\mathfrak{R}\left\{h_{1}^{*}\right\} v_{1}^{k}+j \mathfrak{I}\left\{h_{1}^{*}\right\} v_{1}^{k, \tau}\right) \\
& \approx \sum_{1=1}^{L}\left(\left|h_{1}\right|^{2} d_{k}+\mathfrak{R}\left\{h_{1}^{*}\right\} v_{1}^{k}+j \mathfrak{I}\left\{h_{1}^{*}\right\} v_{1}^{k, \tau}\right)
\end{aligned}
$$

The noise terms $v_{1}^{k}$ and its $\tau$-delayed version $v_{1}^{k, \tau}$ are assumed to be zero mean uncorrelated Gaussian random variables with $\sigma_{v}^{2}=N_{o} / 2$. If the bandwidth of the bandpass filter of Figure 2 is $B$, then it can be shown [12, Eqn. 4.1-57] that the autocorrelation function of $v_{1}^{k}$ is:

$$
\rho_{v v}(\tau)=N_{o} \frac{\sin (\pi B \tau)}{\pi \tau}=4 f_{c} N_{o} \frac{\sin \left(\pi B / 4 f_{c}\right)}{\pi}
$$




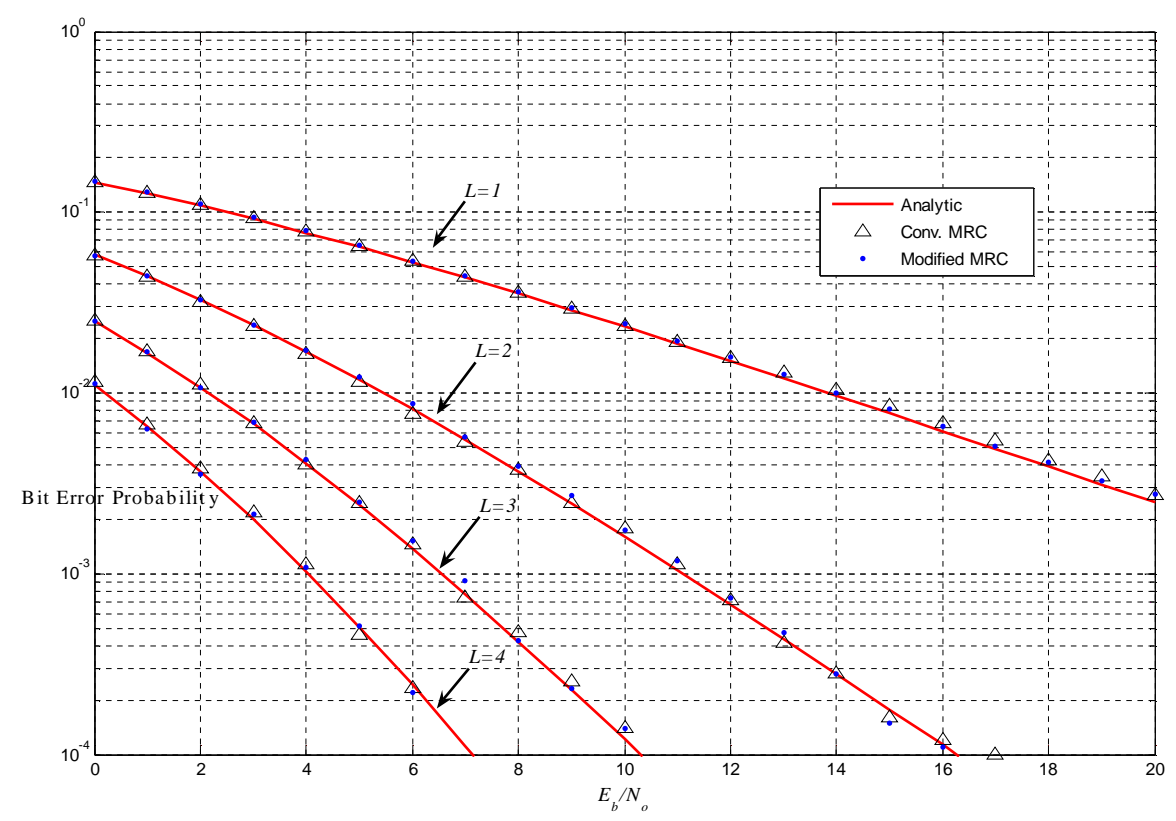

Figure 4 Simulation Results for Binary Phase Shift Keying for various diversity orders.

Thus for any finite bandwidth $\mathrm{B}, \mathrm{v}_{1}^{k}$ and $v_{1}^{k, \tau}$ will be time-correlated, and the cross correlation is given by Equation (16). However, for all practical values of $B$ and $f_{c}$, $B \quad f_{c}$ and hence $\rho_{v v}\left(1 / 4 f_{c}\right) \approx 0$. It is important to note that in the limiting case when $\rho_{v v}\left(1 / 4 f_{c}\right)=1$; Equation (15) becomes exactly the same as equation (6). Therefore, only the assumption of uncorrelated noise samples will be tested via simulations.

Comparing Equations (6) and (15), it is found that the proposed modified MRC scheme achieves full diversity. The validity of the approximations in Equation (14) will be proved by simulations.

\section{Simulation Results}

The analysis presented in Section 3 proves that the diversity scheme depicted in Figure 2 is equivalent to the conventional diversity scheme of Figure 1. Thus, all analytical results found in literature for various types of modulations and various assumptions on the fading characteristics and correlations between antenna branches are applicable. (e.g. [8] and [13] and the references therein). However, the approximations presented in Section 4 warrant a verification of the obtained results. In this section, the validity of these approximations is verified using simulations.

There are basically two validations to be made: The first concerns the properties of the Nyquist pulse shapes giving rise to the approximations in Equation (14). The second 
concerns the correlation properties of the Additive white Gaussian noise processes $v_{1}^{k}$ and $v_{1}^{k, \tau}$.

The former is validated by assuming the use of the steepest pulse shape, in time domain, satisfying Nyquist $1^{\text {st }}$ criterion. Steepest here refers to the maximum decay rate in time domain and hence corresponds to the maximum roll off factor $(r=1)$. Assuming $r_{s} f_{c}$; it follows that $\tau r_{s} \quad 1$ e.g. $\tau r_{s} \approx 0.001$ (this roughly mimics a WiFi System at $2.4 \mathrm{GHz}$ with $11 \mathrm{Mbps}$ data rate), then from the time domain form of the raised cosine pulse shape [14 - Equation 5.7] we have:

$$
\rho_{p p}(\tau)=\frac{\cos \left(\pi r_{s} r \tau\right)}{1-2 r_{s} r \tau} \frac{\sin \left(\pi r_{s} \tau\right)}{\pi r_{s} \tau}=\frac{1}{1-0.002} \frac{\sin (0.001 \pi)}{0.001}=\frac{1}{0.9} \frac{0.0031}{0.0031} \approx 1.0005
$$

and

$$
\begin{aligned}
\rho_{p p}\left(\tau+T_{s}\right) & =\frac{\cos \left(\pi r_{s} r \tau\right)}{1-2 r_{s} r \tau} \frac{\sin \left(\pi r_{s} \tau\right)}{\pi r_{s} \tau}=\frac{\cos (1.001 \pi)}{1-2.002} \frac{\sin (1.001 \pi)}{1.001} \\
& =\frac{-1}{-1.002} \frac{0.0031}{1.0031} \approx 0.003
\end{aligned}
$$

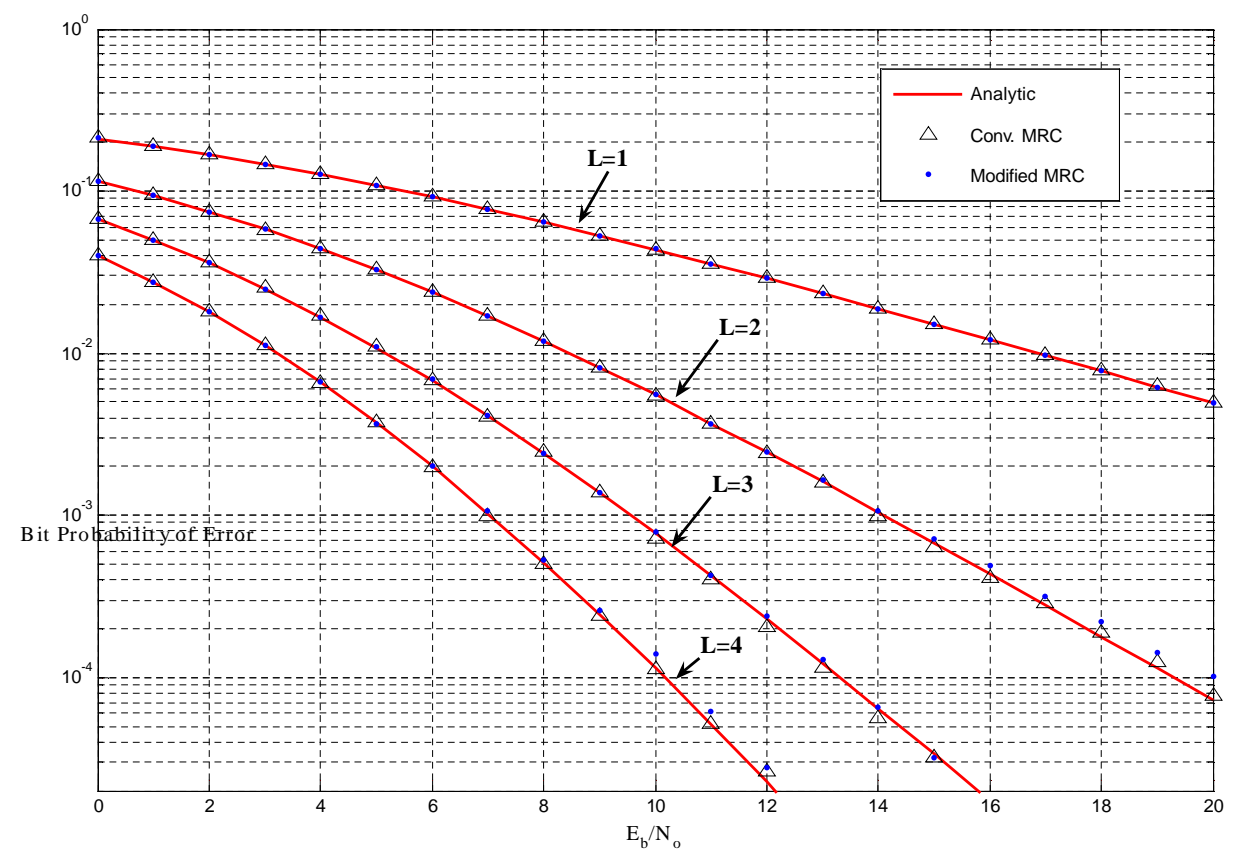

Figure 5 Simulation Results for QPSK for various diversity orders. 
In order to assess and quantify the effect of the approximations, these values are substituted into Equation (13) during simulations instead of using the approximated final form of Equation (15).

The second validation concerning the correlation properties of the discrete noise process $v_{1}^{k}$ and $v_{1}^{k, \tau}$, is made by assuming the two process to be completely uncorrelated (and since they are Gaussian, independent) during simulations. As discussed following Equation (16), this represents the worst case situation since higher correlation ratios improve the approximation.

Figure 4 presents the results for Binary Phase Shift Keying (BPSK) scheme over flat fading channels. All diversity branches are assumed to be independent. The figure compares the results from the proposed modified MRC scheme, obtained from simulating Equation (13) and plotted in dots, to the results of the standard MRC scheme, per Equation (6), plotted in triangles. Perfect channel state information is assumed at the receiver. Both results are show perfect match and coincide with the closed form expression for the probability of error, plotted in red line, given by [13-Equation 9.6]:

$$
P_{b}^{B P S K}=\left(\frac{1-\mu}{2}\right)^{L} \sum_{k=0}^{L-1}\left(\begin{array}{c}
L-1+k \\
k
\end{array}\right)\left(\frac{1+\mu}{2}\right)^{k}
$$

Where $\mu=\sqrt{\bar{\gamma} / 1+\bar{\gamma}}$ and $\bar{\gamma}$ is the average signal to noise ratio per bit.

The figure clearly indicates that the approximations used to arrive at the modified MRC structure of Figure 3-b are valid and cause no loss of performance for all diversity orders considered $L \in[1,4]$.

Similar results are shown for Quadrature Phase Shift Keying (QPSK) modulation in Figure 5. Again, the proposed MRC is compared to the conventional MRC scheme and both are compared in turn with the analytical formula. However, in this case the analytical formula for the bit error probability is given by [12 -Equation 14.4-41]:

$$
P_{b}^{Q P S K}=\frac{1}{2}\left[1-\frac{\mu}{\sqrt{2-\mu^{2}}} \sum_{k=0}^{L-1}\left(\begin{array}{c}
2 k \\
k
\end{array}\right)\left(\frac{1-\mu^{2}}{4-2 \mu^{2}}\right)^{k}\right]
$$

The comparison for all diversity orders shows no loss of performance using the proposed scheme relative to the conventional MRC scheme which requires $L$ matched filters and $L$ RF down converters. 


\section{Conclusions:}

A modified MRC scheme was proposed for use in antenna diversity systems. It provides significant complexity reduction by requiring only one RF down conversion chain for the whole system. The proposed scheme is an approximation of the exact bandpass equivalent of the conventional baseband MRC scheme, which ensures a feasible implementation. Simulation results for BPSK and QPSK wer used to verify the validity of the proposed model. No loss of performance with respect the conventional scheme was reported. MIMO extensions of the proposed scheme are under investigation.

\section{References:}

[1] S. M. Alamouti, "A simple transmit diversity technique for wireless communications" IEEE Journal on Selected Areas in Communications, Vol. 16, No. 8, pp.1451-1458 Oct 1998.

[2] A. Naguib, N. Seshadri, and A Calderbank, "Increasing data rate over wireless channels," IEEE Sig. Processing Magazine, Vol. 17, No. 3, pp. 76-92, May 2000.

[3] P. Pasanen, O. Tirkkonen, "Selection diversity in multiple antenna systems with matrix modulation" Proceedings of International Symposium on Information Theory, p287. ISIT 2004.

[4] J. N. Laneman, D. N. Tse, and G. W. Wornell, "Cooperative diversity in wireless networks: Efficient protocols and outage behavior," IEEE Transactions on Information Theory, vol. 50, no. 11, November 2004.

[5] I. Hammerstrom, M. Kuhn, and A. Wittneben "Cooperative Diversity by Relay Phase Rotations in Block Fading Environments" In proc. of the 5th IEEE Workshop on Signal Processing Advances in Wireless Communications, Lisboa, Portugal, July 11-14, 2004

[6] A. Sendonaris, E. Erkip, and B. Aazhang, "User cooperation diversity-Part I: System description," IEEE Trans. Commun., vol. 51, pp. 1927-1938, Nov. 2003.

[7] D. Brennan, "Linear diversity combining techniques,". Proc. IRE, vol. 47, pp. 1075-1102, June 1959.

[8] D. Smith and T.D. Abhayapala, "Maximal Ratio Combining Performance Analysis in Rayleigh Fading Channels", in IEE Proc. Communications, Vol. 153, Issue 5, pp. 755-761, Oct 2006. 
[9] F.A. Dietrich and W. Utschick. "Maximum Ratio Combining of Correlated Rayleigh Fading Channels with Imperfect Channel Knowledge". IEEE Communications Letters, Vol. 7, No. 9, 419-421, Sept. 2003.

[10] L. Schmitt, T. Grundler, C. Schreyoegg, I. Viering, and H. Meyr, "Maximum ratio combining of correlated diversity branches with imperfect channel state information and colored noise" In proceedings of IEEE 8th International Symposium on Spread Spectrum Techniques and Applications, pp. 859- 863 Sept. 2004.

[11] A. F. Molisch, M. Z. Win, and J. H. Winters, "Reduced-complexity transmit/receive-diversity systems" IEEE Trans.Signal Processing, Vol. 51, No. 11, pp. $2729-2738$ Nov 2003

[12] J.G. Proakis, "Digital Communications," 4th Edition, McGraw-Hill, 2001.

[13] M. K. Simon and M.-S, Alouini, "Digital Communications over Fading Channels, A Unified Approach to Performance Analysis" Wiley, 2000.

[14] K Sam Shanmugam, "Digital and Analog Communication Systems" Wiley 1979.

[15] Sumanasena, A.K.; Chen, N.; Heaton, R. "Low-complexity antenna diversity receiver for ISDB-T system" IEEE 59th Vehicular Technology Conference. VTC 2004 Vol. 1, pp. 22 - 26 Spring 2004. 